\title{
Hidden conformations differentiate day and night in a circadian pacemaker
}

Authors: Jeffrey A. Swan ${ }^{1 *}$; Colby R. Sandate ${ }^{2}$, Archana G. Chavan ${ }^{3}$, Alfred M. Freeberg ${ }^{1}$, Diana Etwaru ${ }^{1}$, Dustin C. Ernst ${ }^{4}$, Joseph G. Palacios ${ }^{1}$, Susan S. Golden ${ }^{4,5}$, Andy LiWang ${ }^{3,4}$, Gabriel C. Lander ${ }^{2} \uparrow$; Carrie L. Partch ${ }^{1,4} \dagger$

\author{
Affiliations: \\ ${ }^{1}$ Department of Chemistry \& Biochemistry, University of California, Santa Cruz, CA 95064 \\ ${ }^{2}$ Department of Integrative Structural and Computational Biology, The Scripps Research \\ Institute, La Jolla, CA 92131 \\ ${ }^{3}$ Department of Chemistry and Biochemistry, University of California, Merced, CA 95343 \\ ${ }^{4}$ Division of Biological Sciences, University of California, San Diego, La Jolla, CA 92093 \\ ${ }^{5}$ Center for Circadian Biology, University of California, San Diego, La Jolla, CA 92093 \\ *These authors contributed equally to this work. \\ $\dagger$ Corresponding authors.
}

15

Correspondence to: cpartch@ucsc.edu, glander@ scripps.edu

Short title: Day/night differences in KaiC structure

One-Sentence Summary: Cryo-EM structures of KaiC in its day and night states reveal the structural basis for assembly of clock regulatory complexes. 


\section{Abstract}

The AAA+ protein KaiC is the central pacemaker for cyanobacterial circadian rhythms.

Composed of two hexameric rings with tightly coupled activities, KaiC undergoes changes in autophosphorylation on its C-terminal (CII) domain that restrict binding of of clock proteins on its N-terminal (CI) domain to the evening. Here, we use cryo-electron microscopy to investigate how daytime and nighttime states of $\mathrm{CII}$ regulate $\mathrm{KaiB}$ binding to $\mathrm{CI}$. We find that the $\mathrm{CII}$ hexamer is destabilized during the day but takes on a rigidified $\mathrm{C}_{2}$-symmetric state at night,concomitant with ring-ring compression. Residues at the CI-CII interface are required for phospho-dependent KaiB association, coupling ATPase activity on CI to cooperative KaiB recruitment. Together these studies reveal how daily changes in KaiC phosphorylation regulate cyanobacterial circadian rhythms. 


\section{Main Text:}

Cyanobacteria possess an internal circadian clock that temporally aligns gene expression with the solar day to maximize photosynthetic output and coordinate integrated metabolic processes (1-3). autophosphorylation in the C-terminal (CII) domain of the hexameric clock protein KaiC (4) that results in the following sequence of post-translational modifications at residues S431 and T432: $\mathrm{S} / \mathrm{T}$ ? S/pT ? pS/pT ? pS/T $(5,6)$. During the day, autophosphorylation is stimulated by another clock protein, KaiA; and at night, compression of the two KaiC rings opens the N-terminal (CI) ring and exposes binding sites that allow a third clock protein, KaiB, to interact with KaiC and sequester KaiA (7). Without KaiA bound to CII, KaiC begins to autodephosphorylate until KaiB and KaiA are released from CI, thus completing the negative feedback loop. Both in vitro and in vivo studies have linked the $\mathrm{S} / \mathrm{T}$ and $\mathrm{S} / \mathrm{pT}$ phosphostates of KaiC with daytime, while the pS/pT and $\mathrm{pS} / \mathrm{T}$ states are associated with the $\mathrm{KaiB}$-associated nighttime state of $\operatorname{KaiC}(5,6)$. The structural basis for formation of the nighttime $\operatorname{KaiABC}$ complex is understood $(8,9)$, however no direct mechanistic link between CII phosphorylation and regulation of KaiB binding has yet been identified.

KaiC can be trapped in its daytime or nighttime state using phosphomimetic substitutions at phase-defining residues (6), referred to herein as KaiC-AE for the daytime variant (S431 AT432E) and KaiC-EA for the nighttime variant (S431E-T432A, see Table S1). Despite stark differences in clock protein association, previous crystallographic studies of the phosphomimetic forms did not reveal structural changes that might explain their distinct biochemical properties (10). Nevertheless, solution biophysical studies have demonstrated overall changes in the shape 
of KaiC throughout the phosphocycle $(11,12)$ as well as changes in intra- and inter-subunit interactions of the adenosine triphosphatase (ATPase) domains (13). Here, we present cryoelectron microscopy (cryo-EM) structures of daytime and nighttime KaiC that reveal new conformations demonstrating how temporal information encoded in CII phosphorylation is transmitted through dynamic structural features to regulate the cyanobacterial circadian clock.

To establish a baseline for KaiB discrimination between the phosphomimetics, we first compared KaiB affinity in the daytime and nighttime-trapped forms of KaiC (Figs. 1A, S1A). We observed $\geq 30$-fold tighter binding of KaiB to nighttime KaiC than the daytime variant, recapitulating previously observed day/night distinction between the KaiC-AE and KaiC-EA phosphomimetics (6). Next, we subjected KaiC-EA and KaiC-AE to comparison by cryo-EM in the presence of a saturating concentration of adenosine triphosphate (ATP) to identify structural differences between the phosphomimetic variants in solution. consistent with previous solution studies where daytime KaiC has exhibited an ‘open’ or destabilized CII hexamer $(13,14)$. Since flexible domains are often susceptible to damaging interactions with the hydrophobic air-water interface during sample preparation, we also structurally characterized daytime $\mathrm{KaiC}$ in the presence of perfluorinated fos-choline, which is known to limit air-water-interface interactions. This resulted in the same $\mathrm{C}_{6}$-symmetric 'extended' conformation previously observed by crystallography in the presence of nonhydrolyzable ATP analogue (10) (Figs. S2, 1C, PDB: 7S67), this time resolved to $\sim 3.8 \AA$. However, in our structure, adenosine diphosphate (ADP) was situated at the CII-CII interfaces, 
suggesting that CII nucleotide state does not influence global conformation in the ground state of daytime KaiC. Notably, the daytime and nighttime KaiC phosphomimetics maintained functional discrimination for KaiB in the presence of fos-choline (Fig S3), consistent with the idea that these open and extended conformations are both present in the solution structural ensemble of daytime KaiC.

In the nighttime phosphomimetic, single particle analysis resulted in two distinct maps with both the $\mathrm{CI}$ and $\mathrm{CII}$ rings resolved (Figs. S4, 1B). Once again, we observed the $\mathrm{C}_{6}$-symmetric 'extended' conformation, this time at $\sim 2.9 \AA$ resolution (PDB: 7S66), as well as a population of particles exhibiting a novel $\mathrm{C}_{2}$-symmetric structure, resolved to $\sim 3.2 \AA$ (PDB: 7S65). This subpopulation of KaiC hexamers has a widened central pore (Fig. 1D-E), coupled with accumulation of ADP at CII-CII interfaces opposite each other in the hexameric ring. KaiC protomers adjacent to these sites adopt a 'compressed' conformation that brings the CI and CII domains together (Fig. 1C, 2A), while the other four protomers remain in the extended conformation. It should be noted that $\mathrm{C}_{2}$-symmetry is rare in hexameric AAA+ proteins, with only a few other examples previously reported (15-17). In contrast to these previously described 'dimer of trimers' states, which each exhibit apo nucleotide pockets at their seam protomers (Fig. S5), the $\mathrm{C}_{2}$-symmetric state of $\mathrm{KaiC}$ is unique in having its seam protomers occupied by ADP, suggesting thermodynamic coupling between CII phosphorylation and nucleotide state (see exhibit changes in accessibility of the pore loop (Fig. S6A), which is in turn linked to CII autophosphorylation (18) (Fig. S6B-C), providing a mechanistic link between KaiA association and nucleotide exchange in CII. 
These observations raise the question: how does ring compression influence KaiB association? In the extended KaiC protomers of both the $\mathrm{C}_{6}$ and $\mathrm{C}_{2}$-symmetric structures, the sidechains of residues K457 and R459 interact directly with the $\gamma$-phosphate of ATP (Fig. 2B). However, at the ADP-occupied CII interfaces, contact with these sidechains is lost (Fig. 2C) and the CII domain on the compressed protomer breaks away from the adjacent CII interface. Computational studies have highlighted the importance of nucleotide interactions at CII interfaces on global conformational dynamics of KaiC (19). Given the apparent coupling between CII nucleotide interactions and the configuration of cis interacting domains in the compressed protomers, we wondered whether introducing an alanine residue in place of K457 would make this state accessible to the daytime variant and influence KaiB association. Consistent with our prediction, the K457A mutation resulted in a $~ 6$-fold increase in KaiB affinity for daytime KaiC (Figs. 2D, S1B), showing that KaiB association is bolstered by mutations that promote the compressed conformer in the daytime state. Likewise, a smaller effect was observed with the same mutant in the nighttime variant, demonstrating that K457A enhances KaiB association via dynamic interactions in the CII nucleotide cleft.

CII nucleotide state and associated ring compression are also linked with changes in secondary structure in the $\mathrm{C}_{2}$-symmetric KaiC structure. In particular, the $\alpha 9$-helix, where the adjacent phase-determining phosphosites S431 and T432 are located, comprises only a single turn in the extended protomers (Fig. 3A), but was lengthened significantly in the ADP-bound, compressed protomers (Fig. 3B). When we inhibited helical elongation by mutating I430 to glycine, we 
observed a $\geq 50$-fold decrease in KaiB affinity (Fig. 3C, S1C), demonstrating that KaiB association is dependent on secondary structure formation induced by the proximal phosphosites.

Lengthening of CII- $\alpha 9$ was also associated with translocation of CII- $\alpha 8$ toward the CI-CII interface in our compressed KaiC structure (Fig. 3D). A recent study highlighted the significance of tyrosine 402 as a key pacemaking residue on CII- $\alpha 8$ (20), with mutants at this position exhibiting extreme changes in CI ATPase activity and correlated circadian period in vivo (e.g., from 15 hours to $>6$ days). Notably, we observed EM density for distinct rotamer conformations of Y402 in the compressed protomers (Fig. S7A) situated near other phase-determining sidechains on the linker connecting CI and CII (21), indicating that dynamic interactions of CI with elements on CII- $\alpha 8$ are key determinants of circadian period for the oscillator.

The translocation of CII- $\alpha 8$ between the extended and compressed conformations (Fig. 3D) appears to regulate biochemical activity of CI via four sequential, highly conserved (Fig. S8), arginine residues in the CI domain known as the 'arginine tetrad' (Fig. 3E) (10). These residues are essential for circadian rhythms, forming a network of electrostatic interactions that join each CI protomer to both clockwise and counter-clockwise neighbors (10, 22) (Fig. S9, Table S2). The arginine tetrad is situated between the CI and CII domains on the tip of the CI- $\beta 9$ hairpin that leads directly into the nucleotide binding pocket of CI (Fig. S9B), where enzymatic activity is required for KaiB association (23). To test whether the arrhythmic phenotype of arginine tetrad mutants $(10)$ is due to disrupted regulation of KaiB binding, we measured KaiB affinity for two arginine tetrad mutants. Affinity was reduced by >10-fold for R216A (Fig. 3C, S1C) and by 6-fold for R217A, highlighting the importance of interdomain regulation for KaiB association. 
The cooperative nature of KaiB binding to the KaiC hexamer is well established $(24,25)$. Given that the arginine tetrad is structurally poised to facilitate both trans and cis protomer regulation, we next wondered whether KaiC-R217A is capable of regulating cooperative recruitment of KaiB. To quantify KaiB cooperativity, we included unlabeled KaiB-I87A, often abbreviated as fold-switched or fsKaiB, as a secondary titrant to stimulate cooperative association of fluorescently labeled KaiB in KaiC binding assays (Fig. S10A) (26). Using this approach, we measured a cooperativity index of $\sim 25 \pm 2$ for KaiC-EA with fsKaiB (Fig. 4A, S10B). Since KaiB takes on the structure of fsKaiB when associated with $\operatorname{KaiC}(8,9,27)$, we interpret this as a 25-fold affinity enhancement for association of subsequent KaiB molecules once the first KaiB molecule binds to the hexamer. The R217A mutant showed a cooperativity index of only $\sim 1.6 \pm$ 0.5 , indicating that trans protomer communication traversing the arginine tetrad is required for KaiB cooperativity. Another arginine tetrad residue, R218, appears to be involved in trans regulation via interaction with the 2' hydroxyl of the nucleotide at the CI-CI interface, sandwiched between the protomers by hydrogen bonds to R218 and H230 (Figs. 4B, S11, Table S2). Substitution of H230 with alanine also diminished cooperativity (Fig. 4A), despite having a relatively small ( 4-fold) effect on KaiB affinity (Fig. 4C, S1D), demonstrating that the CI active site is crucial for synchronizing KaiB recruitment on adjacent protomers.

20 It should be noted that CI residues R218 and H230 have analogs in CII, R451 and H463, that play similar structural roles in trans interactions in the CII domain via the nucleotide (Fig. 2B). Conversely, R215, R216 and R217 of the arginine tetrad are not conserved in CII (Figs. 4D, S8), indicative of their role in CI-CII interdomain regulation in cis. However, the overall architecture 
of the nucleotide binding pocket is strikingly well-conserved between the CI and CII domains of KaiC, and nearly invariant in KaiC across cyanobacteria (Fig. S8). In particular, the CI and CII domains both contain a lysine/arginine pair (K224 and R226 in CI, K457 and R459 in CII). Previously reported high-resolution structures show that K224 changes orientation to coordinate the ultimate nucleotide phosphate at both pre- and post-hydrolysis CI-CI interfaces (Fig. 4B, S11, Table S3), whereas R226 coordinates nucleotide only in the ATP-bound state (22). To determine whether these interactions are required for ATP hydrolysis, we measured the enzymatic turnover rate, $k_{c a t}$, of the R226A, K224A, and H230A mutants (Figs. 4D, S12). We found that the R226A mutation drastically decreased both ATPase activity and KaiB affinity, similar to other catalytically dead variants (23). Conversely, the H230A mutation increased ATPase activity relative to wild type, but diminished KaiB affinity (Fig. 4E), suggesting a more nuanced coupling between ATPase activity and KaiB association than simple correlation. Consistent with this, the K224A mutant had the smallest effect on KaiB affinity and ATPase activity of all the CI active-site mutants tested, but it essentially eliminated cooperativity (Fig. 4A), indicating that $\mathrm{K} 224$ is dispensable for KaiB association on its own protomer, but critical for cooperative regulation of KaiB recruitment in cis.

Given its apparent structural role in sensing nucleotide state at the clockwise CI-CI interface, this result suggests that K224 plays a key role in coupling ATPase activity to cooperativity in KaiB association, thus imparting a switch-like nature to the assembly of protein complexes-a function that has been well documented for other RecA-like AAA+ proteins (28). Notably, we observed total loss of circadian rhythms in vitro with KaiC variants lacking cooperativity (Figs. 4A, 4F), demonstrating that the core oscillator cannot function in the absence of cooperative KaiB recruitment. Furthermore, the KaiC-K224A variant did not support circadian gene 
expression in S. elongatus (Fig. 4G), demonstrating that the presence of additional factors cannot compensate for the loss of $\mathrm{CI}$ nucleotide sensing and cis regulation of KaiB recruitment in vivo.

It should be noted that although ATP hydrolysis in CI is required for KaiB association (23) and ADP-bound CI interfaces are observed in KaiB-bound KaiC structures (8, 9), we never observed ADP-bound CI domains in our cryo-EM structures, indicating that phospho-dependent regulation of KaiB association by CII does not occur simply by trapping the ADP-bound CI state prior to KaiB association. Rather, we propose that ADP is replaced with ATP after hydrolysis unless KaiB association locks the CI domain into the post-hydrolysis state by inhibiting ADP release (Fig. 4H). In this model, S431 phosphorylation regulates the CI domain through elongation of the CII- $\alpha 9$ helix, leading to cis domain compression and translocation of CII- $\alpha 8$ axially towards the arginine tetrad on CI. There, dynamic interactions regulate KaiB association by coupling CI nucleotide state with KaiB occupancy on the same and adjacent CI domains. This work provides a clear mechanistic link between the daily phosphorylation cycle of KaiC and quaternary structural changes that mediate negative feedback in the cyanobacterial circadian pacemaker. Although CI ATPase activity is correlated with circadian period in vivo and in vitro for many KaiC mutants (20-22), no precise chemo-mechanical mechanism has yet been described to explain this. Our results demonstrate that phospho-dependent coupling between the ATPase cycle on CI and cis domain compression influences clock assembly through cooperative KaiB recruitment, explaining why the most extreme period-altering mutations in KaiC are located on CII- $\alpha 8$ or nearby on the linker that connects the CI and CII domains $(20,21)$. These structures and the associated biochemistry pave the way for a comprehensive understanding of 
bioRxiv preprint doi: https://doi.org/10.1101/2021.09.14.460370; this version posted September 15, 2021. The copyright holder for this preprint (which was not certified by peer review) is the author/funder, who has granted bioRxiv a license to display the preprint in perpetuity. It is made available under aCC-BY-NC-ND 4.0 International license.

the post-translational oscillator from both modeling and reverse-engineering perspectives, and underscore the elegant complexity of this sophisticated and primeval timepiece. 

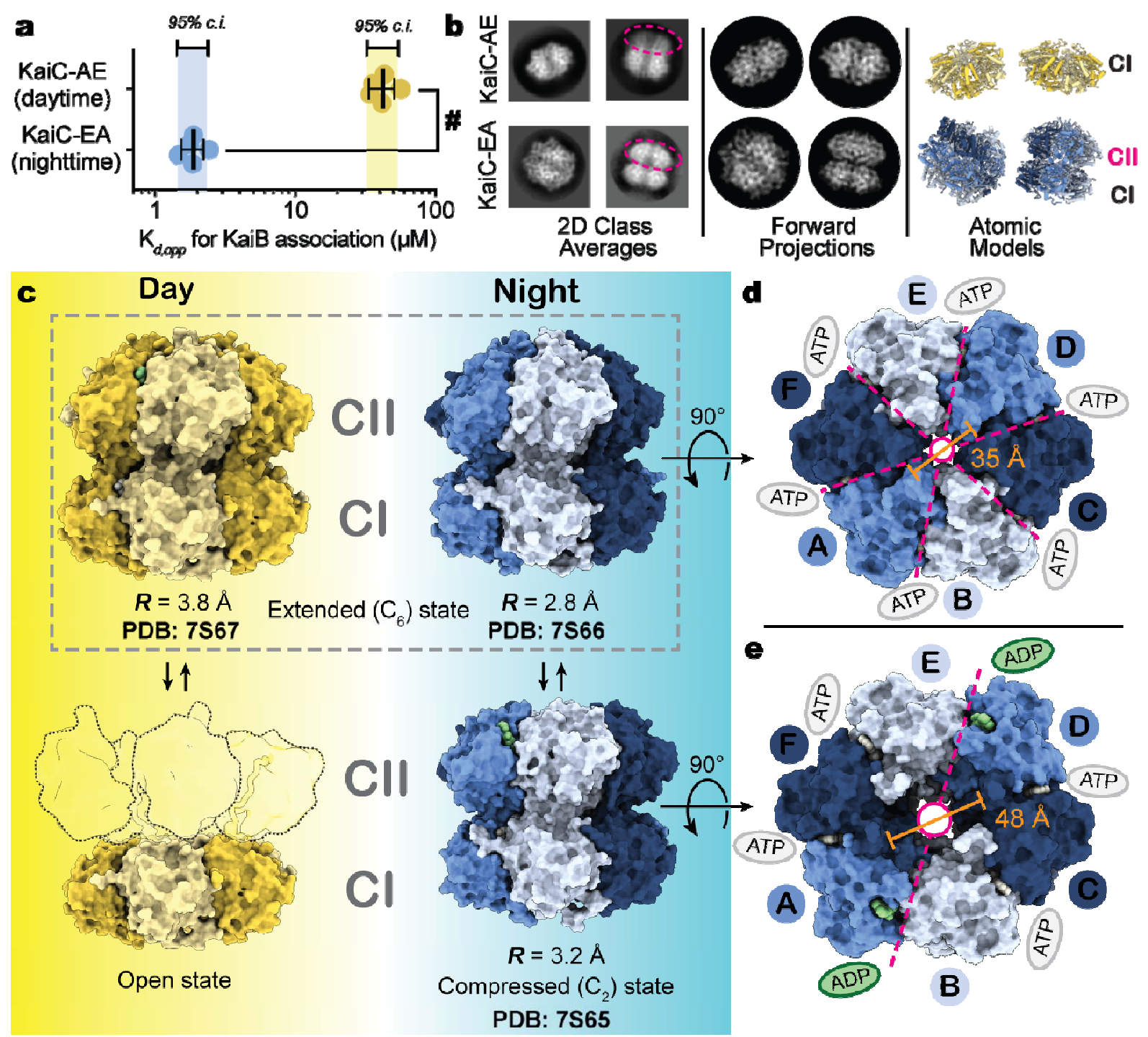

Fig. 1. Daytime and nighttime phosphomimetics confer distinct biochemical activities and

global conformations to KaiC. a) Apparent binding constants $\left(\mathrm{K}_{d, a p p}\right)$ for KaiB association with daytime or nighttime KaiC variants in units of protomer concentration. A hashtag (\#) represents

$P<0.0001$ from an unpaired parametric t-test. Lighter colored regions represent the 95\%

confidence interval for $\mathrm{n}=5$ measurements. b) Reference-free 2-dimensional class averages

from electron micrographs of KaiC phosphomimetics. Dashed ovals indicate the CII rings, where visible, as inferred from forward projections obtained using the atomic models shown. c) Spacefilling depictions of alternate daytime and nighttime KaiC conformations observed by cryo-EM. 
The 'open' daytime state is depicted with outlines to represent the destabilized CII protomers as rigid bodies flexibly tethered to the visible CI hexamer. d-e) Axial view of the extended (d) and compressed (e) nighttime KaiC structures, viewed from the side of the CII. Dashed lines indicate the axes of symmetry with colored ovals denoting nucleotide state in the CII ring. Pore diameters are reported as measured between $\mathrm{C}_{\alpha}$ atoms of $\mathrm{V} 433$ on $\mathrm{A}$ and $\mathrm{D}$ protomers. 

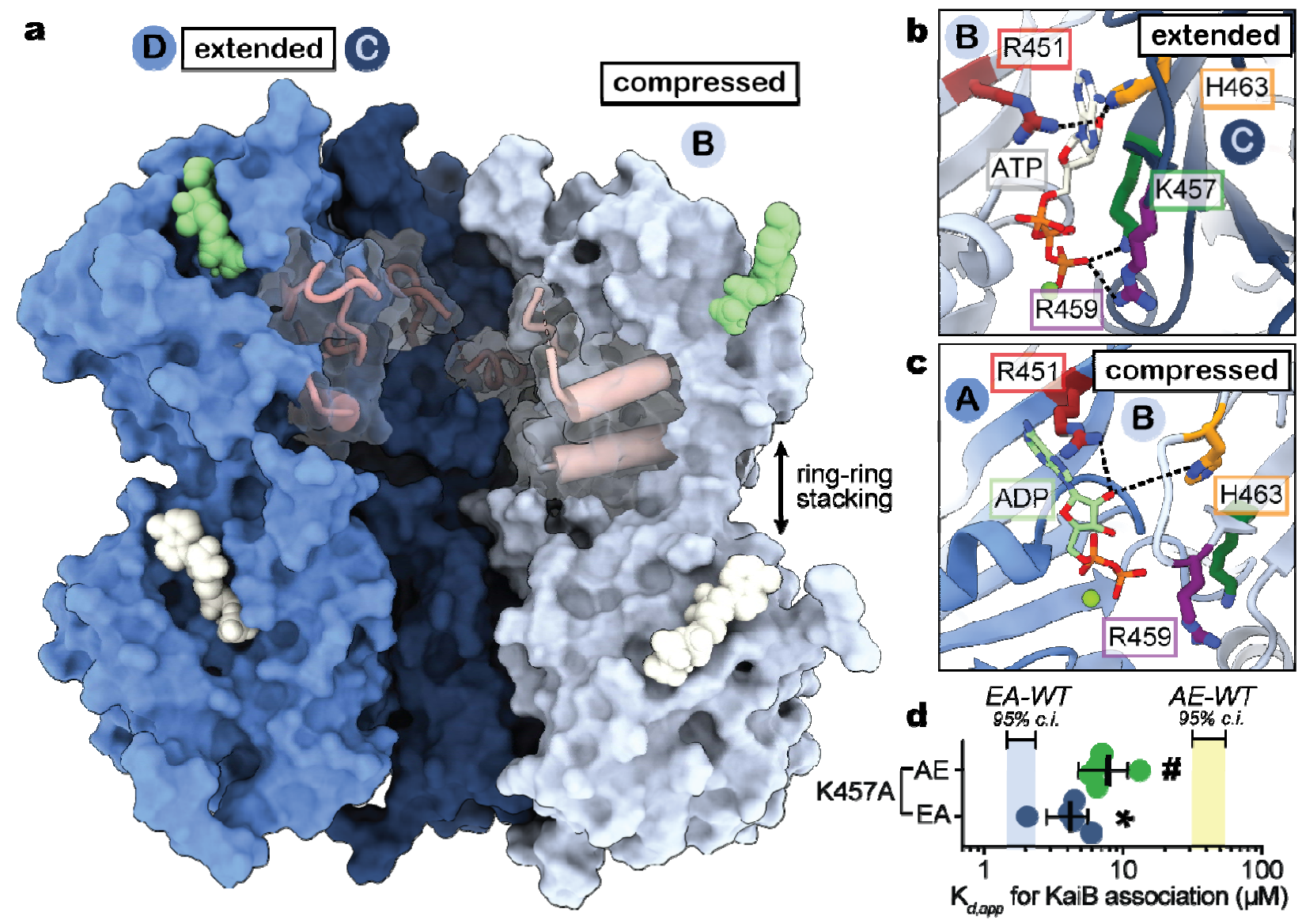

Fig. 2. Nucleotide interactions at CII-CII interfaces govern the transition between

expanded and compressed conformations. a) Surface representation of KaiC protomers in the

$\mathrm{C}_{2}$-symmetric state with CII- $\alpha 8$ and CII- $\alpha 9$ depicted as pink cylinders. b) Closeups of the CII

nucleotide interface in the expanded and (c) compressed KaiC protomer conformations. d) $\mathrm{K}_{d, a p p}$ for the interaction of KaiB with daytime (green) and nighttime (dark blue) KaiC variants bearing the K457A substitution ( $\mathrm{n}=5$ with standard deviation). Symbols (\#, $\mathrm{P}>0.0001 ; *, \mathrm{P}>0.01)$ depict the results of unpaired parametric t-tests comparing each mutant to its respective unsubstituted phosphomimetic. 

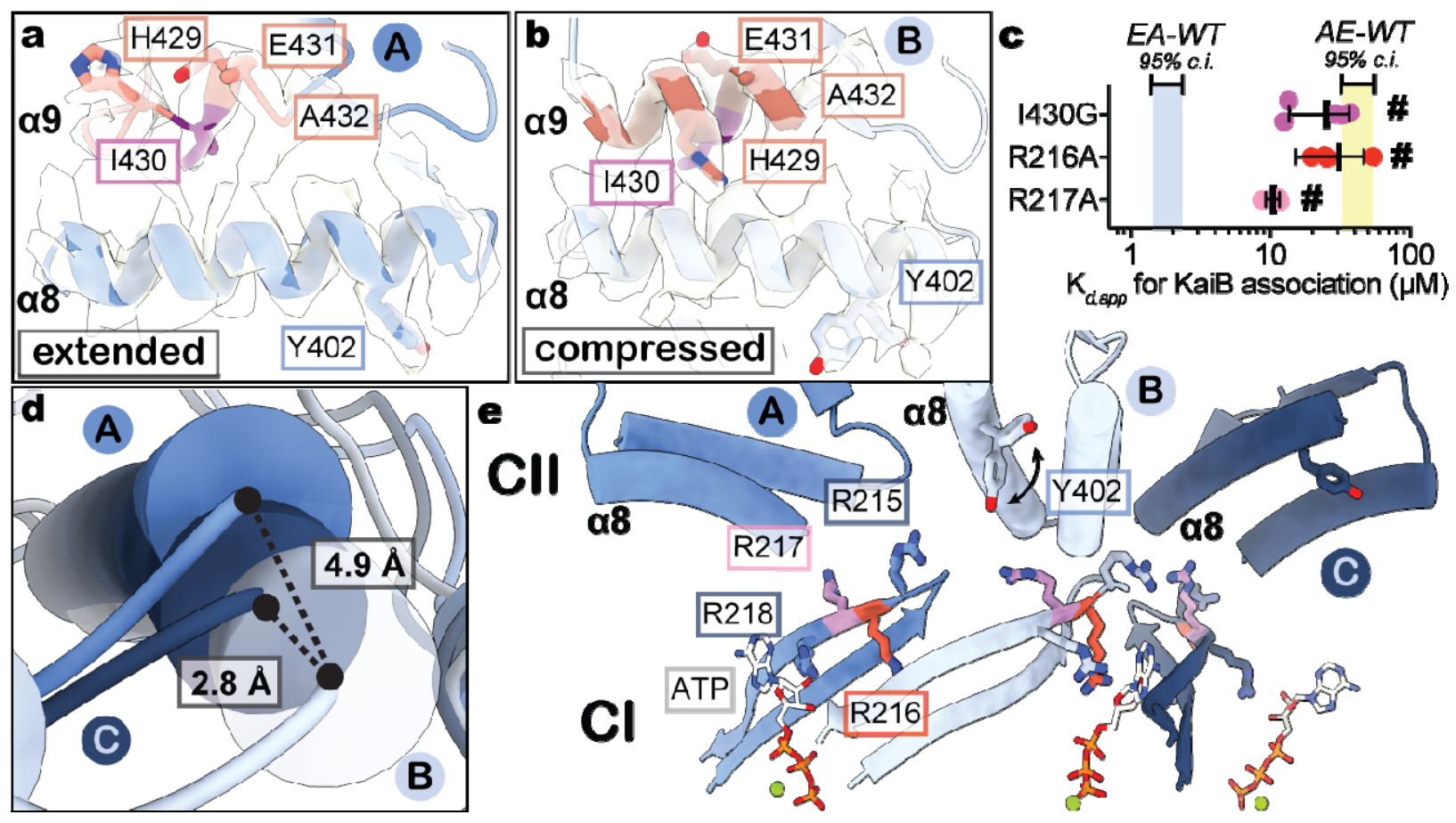

Fig. 3. Compression of the cis-CI-CII interface primes nighttime KaiC for KaiB

association. a-b) EM density and atomic models for residues S424 through T434 (pink) and CII$\alpha 8$ helices in expanded $(\mathbf{a}$, contour $=0.0343))$ and compressed $(\mathbf{b}$, contour $=0.0307)$ protomers arginine tetrad ( $\mathrm{n} \geq 4$ with standard deviation) in nighttime KaiC. Symbols represent one-way ANOVA (\#, P > 0.0001) with Dunnett's multiple comparisons for each mutant against KaiC-EA. d) Cylinder depictions of the CII- $\alpha 8$ helices from the three unique protomer conformations of the compressed hexamer, aligned about their CI domains. e) Proximity of CII- $\alpha 8$ to the arginine tetrad in the $\mathrm{C}_{2}$-symmetric state. 


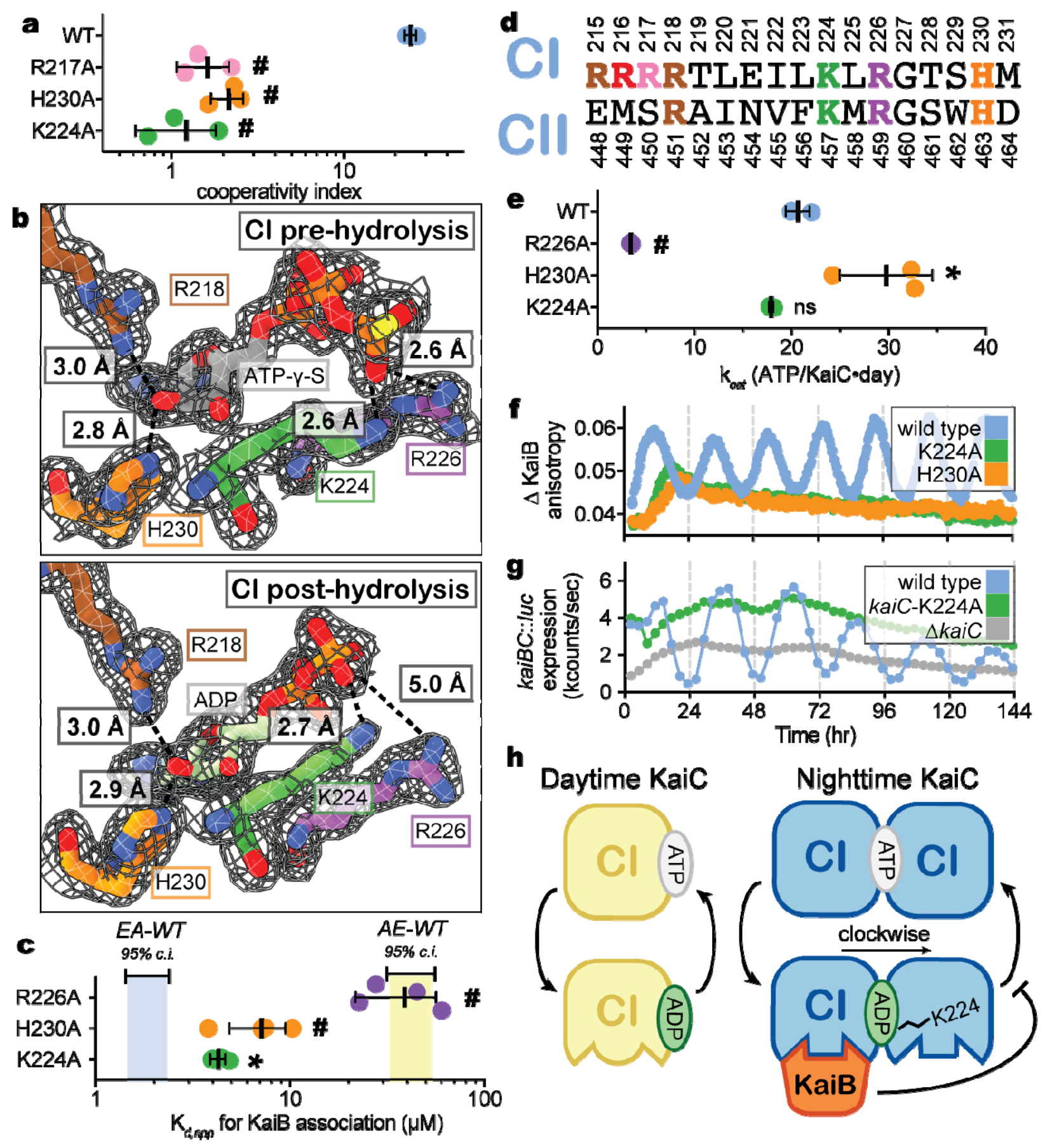

Fig. 4. Interactions in the CI nucleotide binding pocket link ATP hydrolysis to cooperative

KaiB recruitment and circadian rhythms. a) Cooperativity indices for KaiC-EA variants $(\mathrm{n}=$ 3 with standard deviation), determined with fsKaiB as secondary titrant (see text). Symbols represent one-way ANOVA (ns, not significant; *: P > 0.01; \#, P > 0.0001) with Dunnett's multiple comparisons to wild-type KaiC-EA. b) Atomic models and electron density $\left(\mathrm{F}_{\mathrm{c}}-\mathrm{F}_{0}, \sigma=\right.$ 
0.21) for nucleotide interactions from the pre-hydrolysis (PDB 4TL8, top) or post-hydrolysis (PDB 4TLA, chain C, bottom) CI domain crystal structures (22). c) $K_{d, a p p}$ of KaiB binding to nighttime KaiC variants ( $\mathrm{n} \geq 4$ with standard deviation) and statistical analysis as described for (a). d) Alignment of CI and CII protein sequences from S. elongatus. e) Turnover rate constants $\left(k_{c a t}\right)$ for ATP hydrolysis by KaiC-EA variants ( $\mathrm{n}=3$ with standard deviation) with statistical analysis as described for (a). f) In vitro oscillation reactions for KaiC mutants in the presence of KaiA and KaiB. g) Representative bioluminescence timecourses from a luciferase reporter gene driven by $\mathrm{PkaiBC}$ in S. elongatus cultures entrained under 12-h light/dark cycles for 48-hr and subsequently allowed to free-run in constant light $(n=6)$. h) Schematic model for the role of CI ATPase activity in regulation of KaiB association to KaiC. 


\section{References}

1. M. Ishiura et al., Expression of a gene cluster kaiABC as a circadian feedback process in cyanobacteria. Science 281, 1519-1523 (1998).

2. S. Diamond, D. Jun, B. E. Rubin, S. S. Golden, The circadian oscillator in Synechococcus elongatus controls metabolite partitioning during diurnal growth. Proc Natl Acad Sci U S A 112, E1916-1925 (2015).

3. G. K. Pattanayak, C. Phong, M. J. Rust, Rhythms in energy storage control the ability of the cyanobacterial circadian clock to reset. Curr Biol 24, 1934-1938 (2014).

4. F. Hayashi et al., Roles of two ATPase-motif-containing domains in cyanobacterial

circadian clock protein KaiC. J Biol Chem 279, 52331-52337 (2004).

5. M. J. Rust, J. S. Markson, W. S. Lane, D. S. Fisher, E. K. O'Shea, Ordered phosphorylation governs oscillation of a three-protein circadian clock. Science 318, 809812 (2007).

6. T. Nishiwaki et al., A sequential program of dual phosphorylation of KaiC as a basis for circadian rhythm in cyanobacteria. EMBO J 26, 4029-4037 (2007).

7. Y. G. Chang, R. Tseng, N. W. Kuo, A. LiWang, Rhythmic ring-ring stacking drives the circadian oscillator clockwise. Proc Natl Acad Sci U S A 109, 16847-16851 (2012).

8. R. Tseng et al., Structural basis of the day-night transition in a bacterial circadian clock. Science 355, 1174-1180 (2017).

9. J. Snijder et al., Structures of the cyanobacterial circadian oscillator frozen in a fully assembled state. Science 355, 1181-1184 (2017).

10. R. Pattanayek, Y. Xu, A. Lamichhane, C. H. Johnson, M. Egli, An arginine tetrad as mediator of input-dependent and input-independent ATPases in the clock protein KaiC. Acta Crystallogr D Biol Crystallogr 70, 1375-1390 (2014).

11. Y. Murayama et al., Tracking and visualizing the circadian ticking of the cyanobacterial clock protein KaiC in solution. EMBO J 30, 68-78 (2011).

12. A. Mukaiyama et al., Conformational rearrangements of the $\mathrm{C} 1$ ring in KaiC measure the timing of assembly with KaiB. Sci Rep 8, 8803 (2018).

13. Y. G. Chang, N. W. Kuo, R. Tseng, A. LiWang, Flexibility of the C-terminal, or CII, ring of KaiC governs the rhythm of the circadian clock of cyanobacteria. Proc Natl Acad Sci U S A 108, 14431-14436 (2011).

14. K. Oyama, C. Azai, K. Nakamura, S. Tanaka, K. Terauchi, Conversion between two conformational states of KaiC is induced by ATP hydrolysis as a trigger for cyanobacterial circadian oscillation. Sci Rep 6, 32443 (2016).

15. M. Jessop et al., Structural insights into ATP hydrolysis by the MoxR ATPase RavA and the LdcI-RavA cage-like complex. Commun Biol 3, 46 (2020).

16. Q. Z. Ye et al., TRIP13 is a protein-remodeling AAA plus ATPase that catalyzes MAD2 conformation switching. Elife 4, (2015).

17. S. E. Glynn, A. R. Nager, T. A. Baker, R. T. Sauer, Dynamic and static components power unfolding in topologically closed rings of a AAA+ proteolytic machine. Nat Struct Mol Biol 19, 616-622 (2012).

18. Y. I. Kim, G. Dong, C. W. Carruthers, Jr., S. S. Golden, A. LiWang, The day/night switch in KaiC, a central oscillator component of the circadian clock of cyanobacteria. Proc Natl Acad Sci U S A 105, 12825-12830 (2008).

19. L. Hong, B. P. Vani, E. H. Thiede, M. J. Rust, A. R. Dinner, Molecular dynamics simulations of nucleotide release from the circadian clock protein KaiC reveal atomicresolution functional insights. Proc Natl Acad Sci U S A 115, E11475-E11484 (2018). 
20. K. Ito-Miwa, Y. Furuike, S. Akiyama, T. Kondo, Tuning the circadian period of cyanobacteria up to 6.6 days by the single amino acid substitutions in KaiC. Proc Natl Acad Sci U S A 117, 20926-20931 (2020).

21. K. Terauchi et al., ATPase activity of KaiC determines the basic timing for circadian clock of cyanobacteria. Proc Natl Acad Sci U S A 104, 16377-16381 (2007).

22. J. Abe et al., Circadian rhythms. Atomic-scale origins of slowness in the cyanobacterial circadian clock. Science 349, 312-316 (2015).

23. C. Phong, J. S. Markson, C. M. Wilhoite, M. J. Rust, Robust and tunable circadian rhythms from differentially sensitive catalytic domains. Proc Natl Acad Sci U S A 110, 1124-1129 (2013).

24. J. Snijder et al., Insight into cyanobacterial circadian timing from structural details of the KaiB-KaiC interaction. Proc Natl Acad Sci U S A 111, 1379-1384 (2014).

25. R. Murakami et al., Cooperative Binding of KaiB to the KaiC Hexamer Ensures Accurate Circadian Clock Oscillation in Cyanobacteria. Int J Mol Sci 20, (2019).

26. A. Chavan et al., Reconstitution of an intact clock reveals mechanisms of circadian timekeeping. Science (in press), (2021).

27. Y. G. Chang et al., Circadian rhythms. A protein fold switch joins the circadian oscillator to clock output in cyanobacteria. Science 349, 324-328 (2015).

28. J. P. Erzberger, J. M. Berger, Evolutionary relationships and structural mechanisms of AAA+ proteins. Annu Rev Biophys Biomol Struct 35, 93-114 (2006).

29. H. Liu, J. H. Naismith, An efficient one-step site-directed deletion, insertion, single and multiple-site plasmid mutagenesis protocol. BMC Biotechnol 8, 91 (2008).

30. K. Naydenova, M. J. Peet, C. J. Russo, Multifunctional graphene supports for electron cryomicroscopy. Proc Natl Acad Sci U S A 116, 11718-11724 (2019).

31. B. Carragher et al., Leginon: an automated system for acquisition of images from vitreous ice specimens. J Struct Biol 132, 33-45 (2000).

32. G. C. Lander et al., Appion: an integrated, database-driven pipeline to facilitate EM image processing. J Struct Biol 166, 95-102 (2009).

33. S. H. Scheres, RELION: implementation of a Bayesian approach to cryo-EM structure determination. J Struct Biol 180, 519-530 (2012).

34. Y. Z. Tan et al., Addressing preferred specimen orientation in single-particle cryo-EM through tilting. Nat Methods 14, 793-796 (2017).

35. N. R. Voss, C. K. Yoshioka, M. Radermacher, C. S. Potter, B. Carragher, DoG Picker and TiltPicker: software tools to facilitate particle selection in single particle electron microscopy. J Struct Biol 166, 205-213 (2009).

36. K. Zhang, Gctf: Real-time CTF determination and correction. J Struct Biol 193, 1-12 (2016).

37. A. M. Roseman, FindEM--a fast, efficient program for automatic selection of particles from electron micrographs. J Struct Biol 145, 91-99 (2004).

38. E. F. Pettersen et al., UCSF Chimera--a visualization system for exploratory research and analysis. J Comput Chem 25, 1605-1612 (2004).

39. R. Pattanayek et al., Structures of KaiC circadian clock mutant proteins: a new phosphorylation site at T426 and mechanisms of kinase, ATPase and phosphatase. PLoS One 4, e7529 (2009).

40. R. A. Nicholls, M. Fischer, S. McNicholas, G. N. Murshudov, Conformation-independent structural comparison of macromolecules with ProSMART. Acta Crystallogr D Biol Crystallogr 70, 2487-2499 (2014). 
41. P. Emsley, K. Cowtan, Coot: model-building tools for molecular graphics. Acta Crystallogr D Biol Crystallogr 60, 2126-2132 (2004).

42. P. D. Adams et al., PHENIX: a comprehensive Python-based system for macromolecular structure solution. Acta Crystallogr D Biol Crystallogr 66, 213-221 (2010).

43. T. I. Croll, ISOLDE: a physically realistic environment for model building into lowresolution electron-density maps. Acta Crystallogr D Struct Biol 74, 519-530 (2018).

44. V. B. Chen et al., MolProbity: all-atom structure validation for macromolecular crystallography. Acta Crystallogr D Biol Crystallogr 66, 12-21 (2010).

45. C. A. Schneider, W. S. Rasband, K. W. Eliceiri, NIH Image to ImageJ: 25 years of image analysis. Nat Methods 9, 671-675 (2012).

46. F. Hayashi et al., ATP-induced hexameric ring structure of the cyanobacterial circadian clock protein KaiC. Genes Cells 8, 287-296 (2003).

47. J. Heisler, A. Chavan, Y. G. Chang, A. LiWang, Real-Time In Vitro Fluorescence Anisotropy of the Cyanobacterial Circadian Clock. Methods Protoc 2, (2019).

48. J. Ungerer, H. B. Pakrasi, Cpf1 Is A Versatile Tool for CRISPR Genome Editing Across Diverse Species of Cyanobacteria. Sci Rep 6, 39681 (2016).

49. S. R. Mackey, S. S. Golden, Winding up the cyanobacterial circadian clock. Trends Microbiol 15, 381-388 (2007).

50. M. Nakajima, H. Ito, T. Kondo, In vitro regulation of circadian phosphorylation rhythm

of cyanobacterial clock protein KaiC by KaiA and KaiB. FEBS Lett 584, 898-902 (2010).

51. T. Nishiwaki, T. Kondo, Circadian autodephosphorylation of cyanobacterial clock protein KaiC occurs via formation of ATP as intermediate. J Biol Chem 287, 1803018035 (2012).

52. T. Nishiwaki-Ohkawa, Y. Kitayama, E. Ochiai, T. Kondo, Exchange of ADP with ATP in the CII ATPase domain promotes autophosphorylation of cyanobacterial clock protein KaiC. Proc Natl Acad Sci U S A 111, 4455-4460 (2014).

53. N. Puri et al., The molecular coupling between substrate recognition and ATP turnover in a AAA+ hexameric helicase loader. Elife 10, (2021).

54. C. UniProt, UniProt: the universal protein knowledgebase in 2021. Nucleic Acids Res 49, D480-D489 (2021).

55. F. Sievers, D. G. Higgins, The Clustal Omega Multiple Alignment Package. Methods Mol Biol 2231, 3-16 (2021). 


\section{Acknowledgments:}

We thank J.C. Ducom at Scripps Research High Performance Computing for computational support, as well as B. Anderson at the Scripps Research Electron Microscopy Facility for microscopy support.

\section{Funding:}

National Institutes of Health grant R01GM121507 and R35141849 to CLP, R01NS095892 and R21GM142196 to GCL, R35GM118290 to SSG, and R01GM107521 to AL. NSF-CREST: Center for Cellular and Biomolecular Machines at the University of California, Merced (NSFHRD-1547848).

\section{Author contributions:}

Conceptualization: JAS, CRS, GL, CLP

Investigation: JAS, CRS, AGC, AMF, DE, CS, DCE, JGP

Funding acquisition: SSG, AL, GL, CLP

Project administration: JAS, GL, CLP

Supervision: SSG, AL, GL, CLP

Writing - original draft: JAS, CRS, GL, CLP

Writing—review \& editing: JAS, CRS, AMF, SSG, AL, GL, CLP

\section{Competing interests:}

The authors have no competing interests to declare.

\section{Data and materials availability:}

$25 \quad$ KaiC structures have been deposited in the PDB and EMDB with the following codes: EA Compressed State, PDB 7S65 and EMD-24850; EA Expanded State, PDB 7S66 and EMD24851; AE Expanded State, PDB 7S67 and EMD-24852. All data are available in the main text or the supplementary materials

\section{Supplementary Materials}

Materials and Methods

Supplementary Text

Figs. S1 to $\mathrm{S} 12$

Tables S1 to S6

References (29-55)

Data S1 to S3 